\title{
Live Demonstration: Single-Exposure HDR Image Acquisition Based on Tunable Balance between Local and Global Adaptation
}

\author{
Jorge Fernández-Berni, Ricardo Carmona-Galán and Ángel Rodríquez-Vázquez \\ Institute of Microelectronics of Seville (IMSE-CNM) \\ Consejo Superior de Investigaciones Científicas y Universidad de Sevilla \\ C/ Américo Vespucio s/n, 41092, Seville, Spain \\ Contact email: berni@imse-cnm.csic.es
}

\begin{abstract}
This live demonstration showcases a high dynamic range technique that compresses wide ranges of illuminations into the available signal range with a single exposure. In order to accomplish such compression, concurrent sensing-processing takes place at the focal plane, weighing the influence of local and global illumination on each pixel response during the image capture. This process is driven by an on-line analysis of the image histogram that also enables the dynamic accommodation of changing illumination conditions. The proposed technique has been implemented on a prototype smart image sensor achieving a dynamic range of $102 \mathrm{~dB}$.
\end{abstract}

\section{INTRODUCTION}

High Dynamic Range (HDR) [1] continues to be a hot research topic within the image sensor community. No specific approach is capable yet of optimally compensating all the trade-offs involved for every possible application framework considered. A state-of-the-art technique in industry, specially for automotive applications, is split-diode HDR [2]. This approach features dual concurrent exposure, with a large photodiode for high sensitivity and a small one for low sensitivity. The technique showcased in this demo [3] also requires two photodiodes per pixel. However, a single exposure suffices in our case, with the large photodiode sensing the pixel value itself and the small one enabling the concurrent tunable balance between local and global adaptation. As a resut, the HDR image representation is available either for readout or further processing at the focal plane. Vision sensors can clearly benefit from this early representation by exploiting massively parallel image processing from the very beginning of the signal processing chain [4].

\section{Live Demonstration SetuP}

We are using the same prototype image sensor, test board and OpenCV-based software environment described in [5] for this live demo. The test board embedding the prototype and an FPGA is shown in Fig. 1(a). Each image captured by the sensor is grabbed by the FPGA and sent to a PC for the online adjustment of $T_{a d j}$, the parameter encoding the balance between local and global illumination. Its value is sent back in real time to the FPGA that reconfigures the control signals of the sensor chip for the next frame to be captured accordingly.



(a)

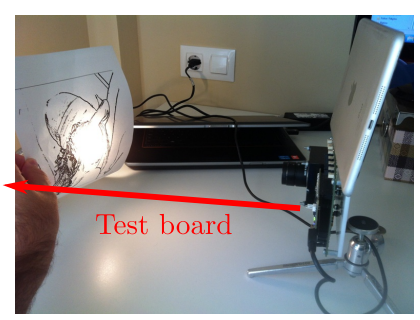

(b)
Fig. 1. Demo setup: (a) test board embedding the prototype image sensor and an FPGA; (b) the visitors will be able to compare the sequence recorded by an iPad mini with the one recorded by our prototype from the same HDR scene.

\section{VISITOR EXPERIENCE}

A HDR scene will be generated by moving a picture of Lena just in front of a lamp, as depicted in Fig. 1(b). The visitors will be able to compare the sequence recorded by an iPad mini with the one recorded by our prototype from this scene. Other HDR scenes could also be set up in the demo booth according to visitors' requirements.

\section{ACKNOWLEDGMENT}

This work has been funded by the Spanish Government through projects TEC2012-38921-C02 MINECO (European Region Development Fund, ERDF/FEDER), Junta de Andalucía through project TIC 2338-2013 CEICE and by the Office of Naval Research (USA) through grant N000141410355.

\section{REFERENCES}

[1] A. Spivak et al., "Wide-dynamic-range CMOS image sensorscomparative performance analysis," IEEE Trans. Electron Devices, vol. 56, no. 11, pp. 2446-2461, 2009.

[2] J. Solhusvik et al., "A comparison of high dynamic range CIS technologies for automotive," in Int. Image Sensor Workshop, 2013.

[3] J. Fernández-Berni, R. Carmona-Galán, and A. Rodríguez-Vázquez, "Single-exposure HDR technique based on tunable balance between local and global adaptation," IEEE Trans. Circuits Syst. II, 2015, accepted, in press.

[4] A. Zarándy, Ed., Focal-plane Sensor-Processor Chips. Springer, 2011.

[5] J. Fernández-Berni et al., "Focal-plane sensing-processing: A powerefficient approach for the implementation of privacy-aware networked visual sensors," Sensors, vol. 14, no. 8, pp. 15 203-15 226, 2014. 